\title{
Neutrophil-to-lymphocyte ratio predicts prognosis of patients with hepatocellular carcinoma: a systematic review and meta-analysis
}

\author{
Shan Lin $^{1,2}$, Shiping $\mathrm{Hu}^{3}$, Yun $\mathrm{Ran}^{3}$, Fenfang $\mathrm{Wu}^{1}$ \\ ${ }^{1}$ Department of Central Laboratory, Shenzhen Hospital, Beijing University of Chinese Medicine, Shenzhen, China; ${ }^{2}$ Institute of Translational \\ Medicine, Shenzhen Second People's Hospital, First Affiliated Hospital of Shenzhen University, Shenzhen University School of Medicine, Shenzhen, \\ China; ${ }^{3}$ Department of Hepatopathy, Shenzhen Hospital, Beijing University of Chinese Medicine, Shenzhen, China \\ Contributions: (I) Conception and design: S Lin, F Wu; (II) Administrative support: S Lin, S Hu; (III) Provision of study materials or patients: S Lin, \\ Y Ran, F Wu; (IV) Collection and assembly of data: S Hu, F Wu; (V) Data analysis and interpretation: S Lin, Y Ran, F Wu; (VI) Manuscript writing: \\ All authors; (VII) Final approval of manuscript: All authors. \\ Correspondence to: Fenfang Wu. Department of Central Laboratory, Shenzhen Hospital, Beijing University of Chinese Medicine, Shenzhen 518100, \\ China. Email: xuchunhua@zju.edu.cn.
}

Background: Neutrophil-to-lymphocyte ratio (NLR) is one predictive factor for poor prognosis of patients with hepatocellular carcinoma (HCC). In response to contradictory data concerning the predictive ability of NLR, we performed a meta-analysis for the determination of its prognostic value in patients with HCC.

Methods: We systematically searched several databases including PubMed, EMBASE, Cochrane Library, Chinese National Knowledge Infrastructure and Wan Fang databases with the updated date of September 21, 2020. Inclusion criteria: RCT studies reporting the prognostic value of the serum levels of NLR in HCC patients receiving treatment were enrolled. Pooled estimates of odds ratio (OR) and diagnostic odds ratio (DOR) were used to assess the prognostic performance of NLR in HCC patients. Overall survival (OS) was the primary outcome and progression-free survival (PFS) was secondary outcomes. Data from studies reporting a hazard ratio and $95 \%$ confidence interval (CI) or a $\mathrm{P}$ value were pooled in a meta-analysis. Furthermore, risk of bias assessment of included studies is specified by Cochrane Risk Bias Assessment Tool. Results: This analysis included 9 studies containing a total of 3,862 HCC patients. High baseline NLR was significantly correlated with poor prognosis or recurrence. The patient-based analysis of pooled estimates was as follows: sensitivity, 0.68 (95\% CI: 0.58-0.77); specificity, 0.73 (95\% CI: 0.61-0.82); DOR, 6.347 (95\% CI: 5.450-7.391). The pooled positive likelihood ratio (PLR) and negative likelihood ratio (NLHR) were 2.5 (95\% CI: 1.8-3.6) and 0.43 (95\% CI: 0.33-0.57). Furthermore, the area under the curve (AUC) of summary receiver operating characteristic (SROC) reflecting the prognostic accuracy was 0.76 (95\% CI: 0.72-0.80). Results obtained from subgroup meta-analyses and overall meta-analyses were accordingly consistent with each other.

Conclusions: Our findings suggested that NLR is an effective prognostic factor for patients with HCC, especially for those from East Asian populations with high incidence. In the future, trials with larger sample sizes and more high-quality evidence are needed to further improve patient outcomes.

Keywords: Hepatocellular carcinoma (HCC); inflammation; neutrophil-to-lymphocyte ratio (NLR); prognostic value; prognosis

Submitted Nov 10, 2020. Accepted for publication Feb 23, 2021.

doi: $10.21037 /$ tcr-20-3237

View this article at: http://dx.doi.org/10.21037/tcr-20-3237 


\section{Introduction}

As the fifth most common malignancy worldwide, hepatocellular carcinoma (HCC) ranks third in mortality rate among cancers (1). In the Asia Pacific region, HCC is a major public health problem because of the relatively high incidence of viral hepatitis (2). It is the second most common cancer in China and the third most common in Korea, and it is insidious and progresses rapidly $(3,4)$. Currently, the overall survival (OS) of HCC is satisfactory due to recent improvements in clinical treatment (5). For instance, surgical resection of HCC is not only reliable and effective but also has a low associated mortality rate (6). Nevertheless, HCC prognosis remains poor. Death after curative resection is mainly attributed to tumor recurrence (7). The recurrence of HCC originates from either de novo tumors arising from the remnant liver or intrahepatic metastasis of the primary tumor (8). To date, the identified risk factors suggest that late recurrence primarily arises from continuous liver disease, whereas early recurrence is largely related to the characteristics of invasive tumors $(8,9)$. Therefore, prognostic assessment of HCC is pivotal for improving the clinical outcomes of patient.

It is commonly known that HCC generally develops from chronic inflammation and cirrhosis (10). The inflammatory responses and immune status of HCC patients have an influence on survival and recurrence after treatment (11). Recently, accumulating evidence has shown that poorer prognosis is correlated with the presence of systemic inflammation in cancer patients (12-16). An elevated level of inflammation, including neutrophil to lymphocyte ratio (NLR), Glasgow Prognostic Score (GPS), C-reactive protein to albumin ratio and platelet to lymphocyte ratio (PLR), have been indicated to be associated with poor survival in HCC patients. Nevertheless, NLR might be more readily available and inexpensive objective compared with the other prognostic indexes that could be used in daily oncologic clinical practice. Moreover, neutrophil-to-lymphocyte ratio (NLR) has been reported as a dependable indicator to monitor and evaluate systemic inflammatory reactions (17). Furthermore, NLR can be repeatedly and easily measured using peripheral blood. Baseline NLR has been reported as a prognostic marker or a valuable factor in many kinds of cancers, including renal cancer (18) and HCC (19). Previous studies investigating its prognostic value in HCC patients undergoing specific treatments indicate that pretreatment NLRs are predictors of tumor recurrence and survival in HCC patients $(20,21)$. However, the exact function of NLR in HCC patients is controversial among studies as a result of many elements, such as variances in sample size, study designs, regional differences, and so on (22). Some studies report a strong correlation between higher NLR and poorer prognosis, while others do not $(18,23)$. It was demonstrated that mutually contradictory data have arisen with regard to the predictive ability of NLR for HCC prognosis, especially in patients from East Asia which has high incidence of HCC (19). We thus conducted a meta-analysis to acquire a systematic and thorough understanding of the prognostic value of NLR in HCC patients.

To the best of our knowledge, few studies have systematically concentrated on the prognostic value of NLR in HCC patients, especially in those from a region with a high morbidity of this disease. In this study, we aimed to illuminate the prognostic value of NLR in HCC patients and determine whether the prognostic accuracy can be increased in a higher HCC-risk region. We present the following article in accordance with the PRISMA reporting checklist (available at http://dx.doi.org/10.21037/tcr-20-3237).

\section{Methods}

\section{Data sources and searches}

A systematic search of several databases including PubMed, EMBASE, and the Cochrane Library in English, Chinese National Knowledge Infrastructure (http://www.cnki. net/) and Wan Fang databases in Chinese (http://www. wanfangdata.com.cn) was performed from inception to September 21, 2020. The search terms used were as follows: ("liver cancer"[Mesh], OR "hepatoma"[Mesh], OR "hepatic carcinoma"[Mesh], OR "hepatocellular carcinoma"[Mesh]), and ("neutrophil to lymphocyte ratio", "neutrophil lymphocyte ratio", OR "neutrophil-to-lymphocyte ratio", OR "inflammatory markers"). Abstracts with complete result sections were included in this study. The bibliography of retrieved articles was manually checked for additional references. This meta-analysis was carried out in accordance with the PRISMA (Preferred Reporting Items for Systematic Reviews and Meta-Analysis) statement issued (Checklist file) $(24,25)$. The present meta-analysis was also submitted to PROSPERO (The International Prospective Register of Systematic Reviews; no. 156404).

\section{Study selection}

All citations were reviewed in turn. Full texts of potentially 
relevant articles were retrieved by titles or abstracts, and eligible studies were determined through independent examinations by 2 investigators (Shan Lin and Shiping $\mathrm{Hu}$ ). Disagreements on eligibility were settled through discussion with an arbitrator (Fenfang $\mathrm{Wu}$ ). Studies that clearly met the following inclusion criteria were considered: (I) participants were aged $\geq 18$ years old for human studies; (II) serum levels of NLR were measured prior to formal treatment; (III) sample size was greater than 30; (IV) randomized controlled trials (RCTs) were observational in nature; (V) sufficient data of true-positives (TP), falsepositives (FP), false-negatives (FN), and true-negatives (TN) for calculating the predictive ability of NLR in HCC patients were provided. Studies were eliminated if they met the following exclusion criteria: (I) only animal or in vitro study was performed; (II) information about prognostic accuracy was lacking in a control or experimental group; (III) article type was a review, commentary, poster, letter, supplementary issue, or editorial; (IV) duplicate data were present or information was insufficient.

\section{Data extraction}

Data from each trial were extracted by 2 reviewers (Shan Lin and Shiping $\mathrm{Hu}$ ) independently. All discrepancies or disagreements between reviewers were discussed with and evaluated by a third investigator (Fenfang Wu) until an agreement was reached. Prespecified data from each article included the request for documentation and recalculation of variables as follows: first author, year of publication, type of publication, study design, regions, sample size (male), enrollment period, median age (years), area under the curve (AUC) [95\% confidence interval (CI)], baseline NLR cutoff, sensitivity, and specificity.

\section{Quality assessment}

Cochrane Risk of Bias Assessment Tool was adopted for quality assessment of the articles by 2 independent reviewers (Shan Lin and Shiping $\mathrm{Hu}$ ) (26). This tool consists of 6 domains: random sequence generation, allocation concealment, blinding, incomplete outcome data, selection outcome reporting, and other resources of bias. "Risk of bias" was evaluated for all 6 domains and "concerns regarding applicability" was assessed with each item judged to be "yes", "no", or "unclear".

The quality of eligible studies was evaluated by
Newcastle-Ottawa Scale (NOS) (27). On the basis of selection, comparability, and exposure, the estimations of study quality were judged using a star-rating system with a maximum of 9 stars. The quality of each trial was defined as poor with 0-3 stars, fair with 4-6 stars, and good with 79 stars. Quality assessment of NOS was performed according to a previous study with some modifications (21).

\section{Statistical analysis}

Stata version 12.0 (StataCorp, College Station, TX, USA) software was used for all statistical analyses of TP, TN, $\mathrm{FP}$, and FN rates for each test in each study and in the assessment of sensitivity, specificity, positive likelihood ratio (PLR), negative likelihood ratio (NLHR), and diagnostic odd ratio (DOR) for each eligible study. A $\mathrm{P}$ value $<0.05$ for $\mathrm{Q}$ statistic and a $\mathrm{I}^{2}$ value $>50 \%$ for $\mathrm{I}^{2}$ statistic were considered to indicate statistically significant heterogeneity (28). The $\mathrm{I}^{2}$ index was used to indicate the degree of heterogeneity among multiple studies with $\mathrm{I}^{2}$ values of $<25 \%, 25-50 \%$ and $>50 \%$, respectively regarded as modest, moderate, and substantial. A random-effects model was adopted when heterogeneity was substantial $\left(\mathrm{I}^{2}>50 \%\right)$ (29). Hardy-Weinberg equilibrium (HWE) in controls of each study was examined by Pearson's $\chi^{2}$ test with a $\mathrm{P}$ value $<0.05$ was considered statistically significant (30).

Summary receiver operator characteristic (SROC) curves and forest plots of pooled sensitivity and specificity were conducted to evaluate the predictive performance of NLR in HCC patients which was measured by calculating AUC as a summary index (31). Moreover, subgroup analyses were performed on a regional or geographic basis. At last, possible publication biases were detected by Begg's and Egger's tests with a $\mathrm{P}$ value $<0.05$ considered statistically significant $(32,33)$.

\section{Results}

\section{Literature search}

Initially, the electronic search yielded 521 potentially relevant studies, of which 276 were excluded after removing duplicates in the databases. A total of 52 were excluded because of obvious irrelevance based on titles or abstracts. The remaining 224 full-text manuscripts were assessed for eligibility, of which 167 were excluded as they failed to meet the requirements of data extraction, and a further 48 were 
eliminated as they did not match the eligibility criteria. Consequently, 9 articles were eligible and included. The above 9 studies included a total of 3,862 patients for metaanalysis of predictive value of NLR for HCC prognosis. The stepwise screening of included studies is shown in

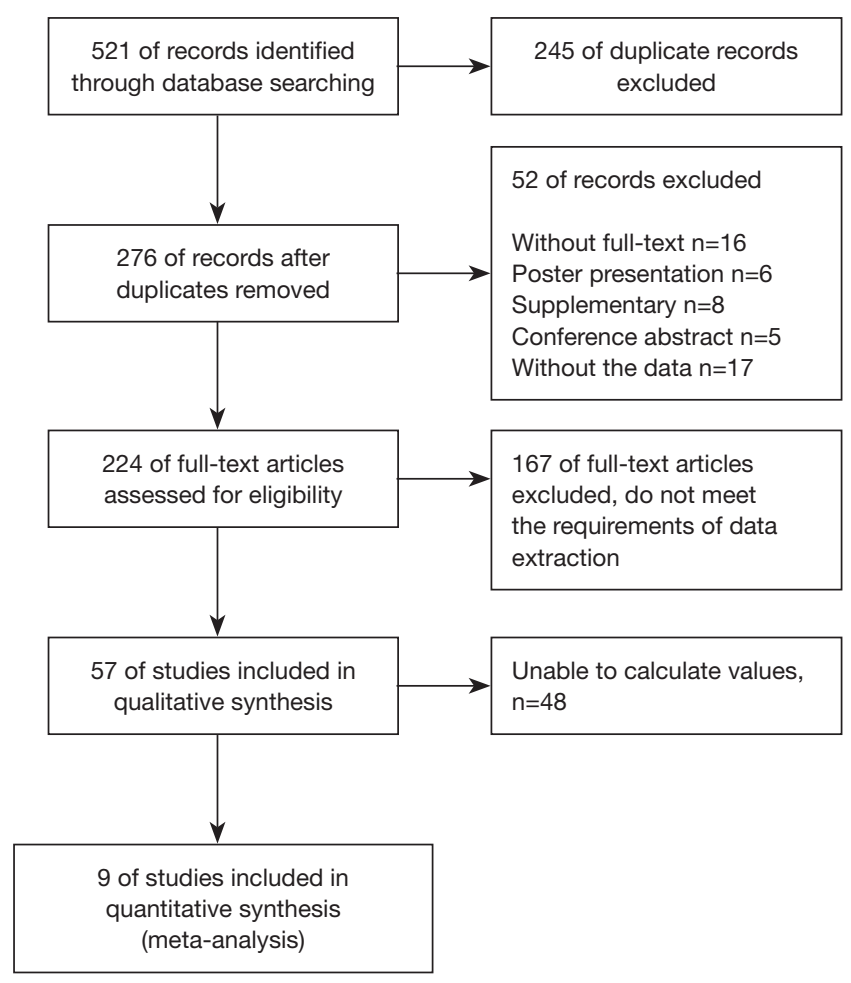

Figure 1 The selection process of the studies included in the meta-analysis. detail in Figure 1.

\section{The characteristics and quality of the included studies}

All of the 9 included studies were written in English. For quality assessment of included studies, baseline data were extracted and are exhibited in Table 1. All of these 9 studies were all from East Asian regions, with 7 from mainland China (34-40), 1 from Korea (41), and 1 from Taiwan (42). All these studies were single-center trials published from 2012 to 2018. The 9 observational studies involved 3,862 HCC patients in total, of which 3,491 were from mainland China, 213 from Korea, and 158 from Taiwan. The predictive performance of NLR for the prognosis of HCC patients is summarized in Table 2. The AUCs of the included studies ranged from 0.606 to 0.855 , and the cut-off values ranged from 1.505 to 2.979. Meanwhile, the sensitivity and specificity of the included studies were calculated or provided, and ranged from 0.301 to 0.840 and from 0.470 to 0.887 , respectively.

\section{Assessment of methodological quality and publication bias}

All studies were clearly defined by eligibility criteria and reasons for patient exclusion. The quality of each included study was assessed by Cochrane Risk of Bias Assessment Tool, and all of them had high Cochrane scores $(\geq 10)$. Overall quality of included trials was moderate. The outcome of Cochrane evaluation was shown in Figure 2.

Subsequently, assessment of publication bias was conducted by funnel plot, as shown in Figure $3 A$, indicating that neither

Table 1 Main characteristics of the studies enrolled in the meta-analysis

\begin{tabular}{llllcccc}
\hline Author [year] & Type of publication & Study design & Regions & Sample size [male] & Enrollment period & \multicolumn{2}{c}{ Median age [years] } \\
\hline Chen [2012] & Full-text & Retrospective & Taipei, Taiwan & $158[95]$ & $2003.07-2010.12$ & $65.7[31.8-82.8]$ & 8 \\
Du [2019] & Full-text & Retrospective & Xi'an, China & $230[174]$ & $2000.01-2012.12$ & $44[20-66]$ & 7 \\
Gao [2015] & Full-text & Retrospective & Beijing, China & $825[690]$ & $2008.10-2012.05$ & $54.5[25-75]$ & 8 \\
Hu [2016] & Full-text & Retrospective & Suwon, Korea & $213[166]$ & $2001.03-2011.12$ & $53[20-79]$ & 8 \\
Hu [2018] & Full-text & Retrospective & Beijing, China & $545[442]$ & $2013.07-2016.07$ & 56.91 & 7 \\
Li [2014] & Full-text & Retrospective & Beijing, China & $506[420]$ & $2005.04-2014.04$ & $59.2[28-85]$ & 8 \\
Liu [2016] & Full-text & Retrospective & Nanjing, China & $223[189]$ & $2004.07-2011.04$ & $54[21-82]$ & 7 \\
Liu [2017] & Full-text & Retrospective & Chengdu, China & $760[643]$ & $2007.01-2013.12$ & $56.5[19-89]$ & 7 \\
Tan [2018] & Full-text & Retrospective & Qingdao, China & $402[299]$ & $2008.09-2017.05$ & $51.7[18-92]$ & 8 \\
\hline
\end{tabular}

NR, no result; NOS, Newcastle-Ottawa Scale. 
Table 2 Predictive value of NLR to predict HCC in individual studies

\begin{tabular}{|c|c|c|c|c|c|c|c|c|c|}
\hline Study & AUC & $95 \% \mathrm{Cl}$ & Cut-off value (ng/mL) & Sensitivity (\%) & Specificity (\%) & \multicolumn{4}{|c|}{ Number of patients } \\
\hline Chen [2012] & 0.630 & $0.520-0.720$ & 2.400 & 0.730 & 0.470 & 59 & 41 & 22 & 36 \\
\hline Du [2019] & 0.625 & $0.527-0.732$ & 2.270 & 0.639 & 0.653 & 57 & 49 & 32 & 92 \\
\hline Gao [2015] & 0.811 & NR & 2.700 & 0.662 & 0.848 & 220 & 75 & 112 & 418 \\
\hline $\mathrm{Hu}$ [2018] & 0.738 & $0.699-0.774$ & 2.979 & 0.539 & 0.858 & 199 & 25 & 170 & 151 \\
\hline Li [2014] & 0.824 & NR & 2.140 & 0.780 & 0.690 & 143 & 100 & 40 & 223 \\
\hline Liu [2016] & 0.606 & NR & 2.750 & 0.301 & 0.887 & 43 & 9 & 100 & 71 \\
\hline Liu [2017] & 0.664 & $0.630-0.698$ & 2.200 & 0.752 & 0.545 & 415 & 95 & 137 & 113 \\
\hline
\end{tabular}

NR, no result; NLR, neutrophil to lymphocyte ratio; HCC, hepatocellular carcinoma; AUC, area under the curve; Cl, confidence interval; TP, true-positive; FP, false-positive; FN, false-negative; TN, true-negative.

significant threshold effect nor significant asymmetry was observed. In other words, this meta-analysis had no obvious publication bias. Therefore, it seems unlikely that our findings would be greatly changed by unpublished studies.

\section{NLR for predicting prognosis in patients with HCC}

There were 9 sets of data extracted from 9 eligible studies (Table 2), including AUC, 95\% CI: various optimal cut-off values of NLR, sensitivities, and specificities, as well as TP, FP, FN, and TN values. The predictive value of NLR as a biomarker for prognosis of HCC patients was inspected in 9 studies in a total number of 3,862 participants. The pooled data of these studies are summarized in Table 2. In the performance estimates of NLR, the pooled sensitivity was 0.68 (95\% CI: 0.58-0.77) (Figure 3B), specificity was 0.73 (95\% CI: 0.61-0.82) (Figure 3C), PLR was 2.5 (95\% CI: 1.8-3.6), and NLHR was 0.43 (95\% CI: 0.33-0.57). The pooled DOR was 6.347 (95\% CI: 5.450-7.391) according to the random effects model. The AUC of SROC for prognostic accuracy summary was 0.76 (95\% CI: 0.72-0.80; Figure 3D). A summary of the predictive value of NLR for the prognosis of HCC patients is shown in Figure 4. We concluded that high baseline NLR was prominently related to poor prognosis or recurrence.

\section{Subgroup analysis}

Subgroup analysis was performed and is summarized in
Table 3. On the basis of comparisons of DOR and AUC, NLR was variable to some extent regarding prognostic value in HCC patients. For subgroup regional analysis, the DOR and AUC of NLR in mainland China were significantly higher than those in Korea (DOR, 7; AUC, 0.79 vs. DOR, 3; AUC, 0.64) and Taiwan (DOR, 7; AUC, 0.79 vs. DOR, 2; AUC, 0.63 ), indicating a better prognostic value of NLR in HCC patients from mainland China than in those from Korea and Taiwan. For subgroup geographic analysis, NLR showed a much higher prognostic value in northern regions than in in southern regions (DOR, 8; AUC, $0.80 v s$. DOR, 3; AUC, 0.69). Interestingly, it was quite obvious that the prognostic value of NLR varies significantly across the North-South geographic boundary of East Asia.

\section{Analysis of publication bias and beterogeneity}

Heterogeneity analysis and SROC were performed. There was no "shoulder arm" pattern seen in the SROC space, suggesting an absence of threshold effect. The publication bias, which was detected by Begg's funnel plot and Egger's test, was at a very low level of probability (Figure 5). Furthermore, meta-regression and subgroup analysis were performed to identify other possible explanations of heterogeneity. Geography (North: $\mathrm{I}^{2}=88.8 \%, \mathrm{P} \leq 0.001$ or South: $\left.\mathrm{I}^{2}=0, \mathrm{P}=0.532\right)$ may be mainly responsible, while the region (mainland China: $\mathrm{I}^{2}=90.1 \%, \mathrm{P} \leq 0.001$, Korea or Taiwan) may not be a source of heterogeneity for the predictive value of NLR. 

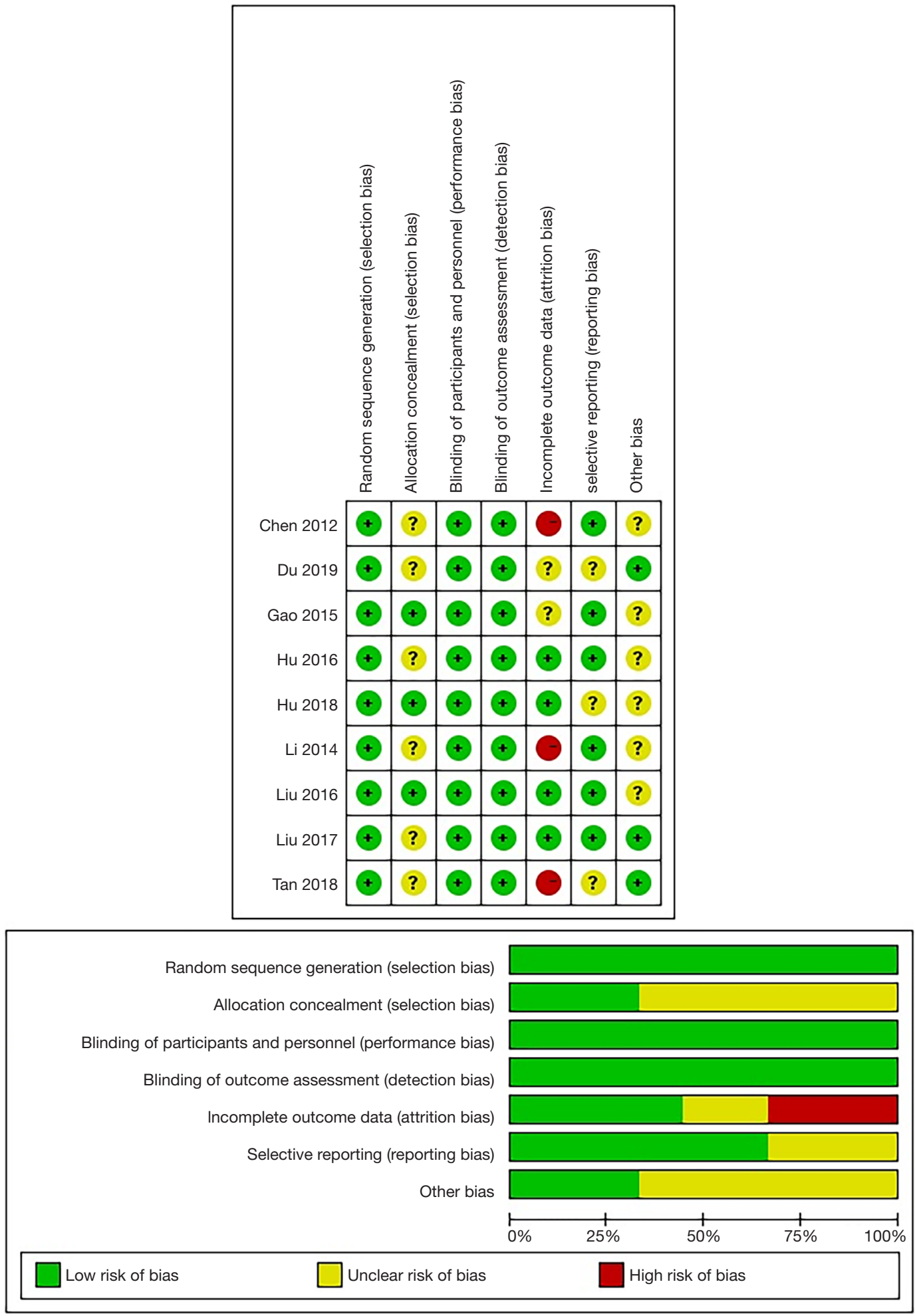

Figure 2 Quality assessment of included eligible studies using Cochrane Risk Bias Assessment Tool. 
A

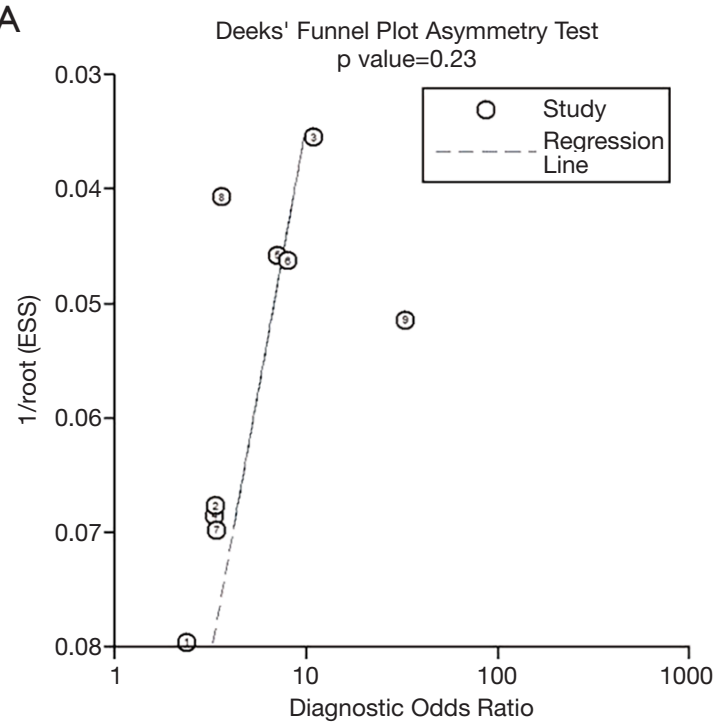

C

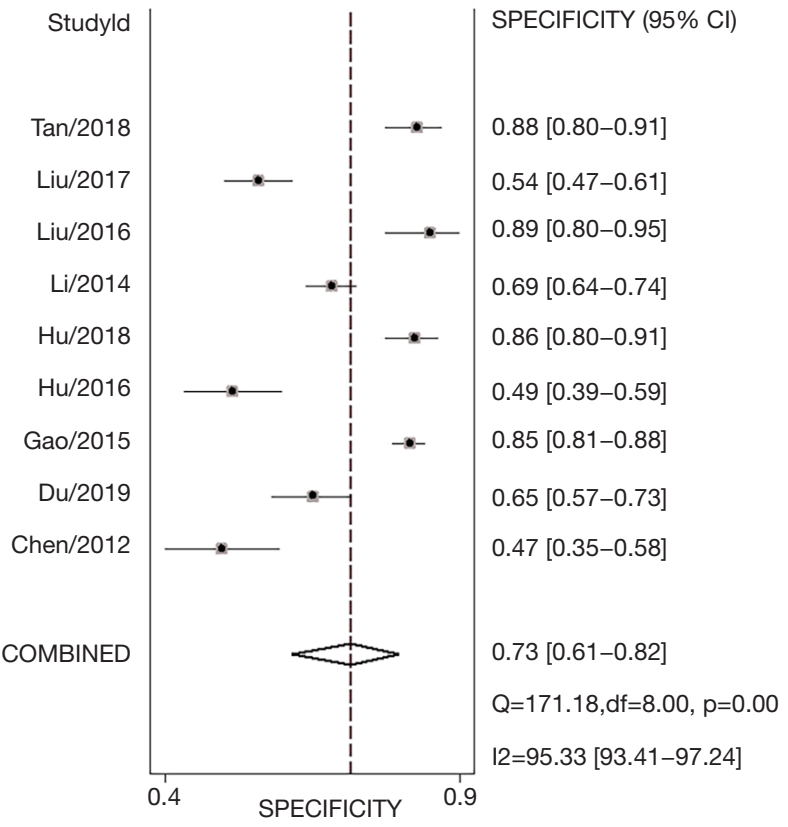

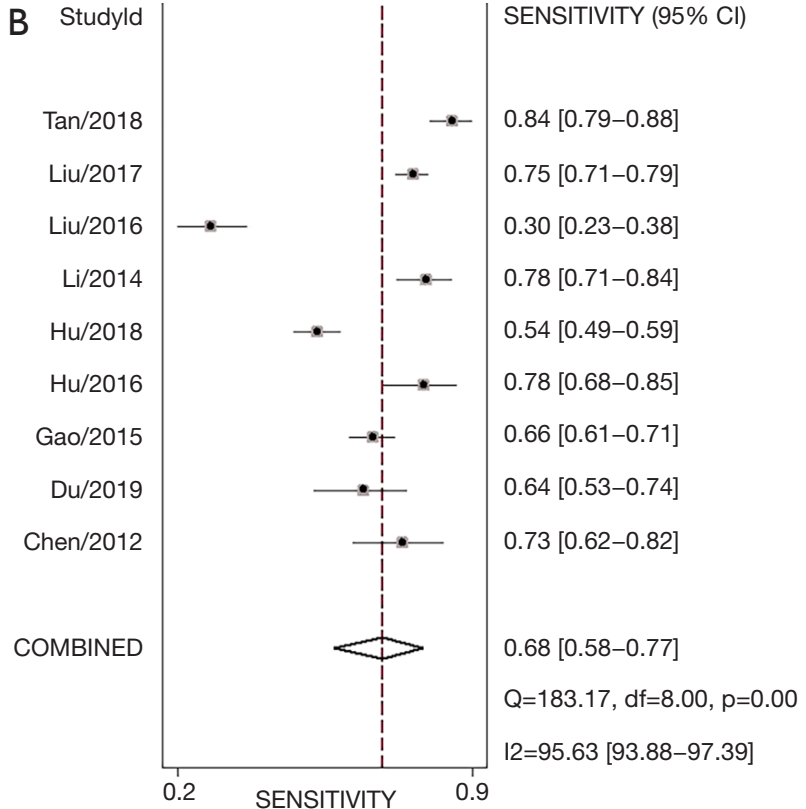

$\mathrm{D}$

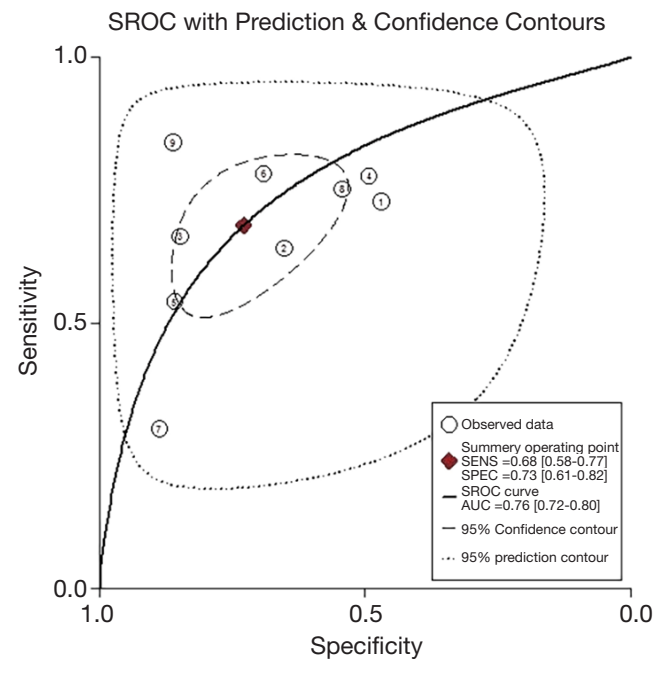

Figure 3 Forest plots of sensitivity, specificity, AUC, and Deeks funnel plot of NLR for predicting prognosis of HCC. (A) Funnel plot. (B) Sensitivity. (C) Specificity. (D) AUC. AUC, area under the curve; NLR, neutrophil-to-lymphocyte ratio; HCC, hepatocellular carcinoma.

\section{Discussion}

As a conventional inflammatory marker, the prognostic function of NLR in HCC patients has been investigated in recent years. In this study, the exact relationship between increased NLR levels and clinical outcomes of HCC was comprehensively and systematically determined in HCC patients, especially in those from East Asia. We mainly investigated the association between high baseline NLR 


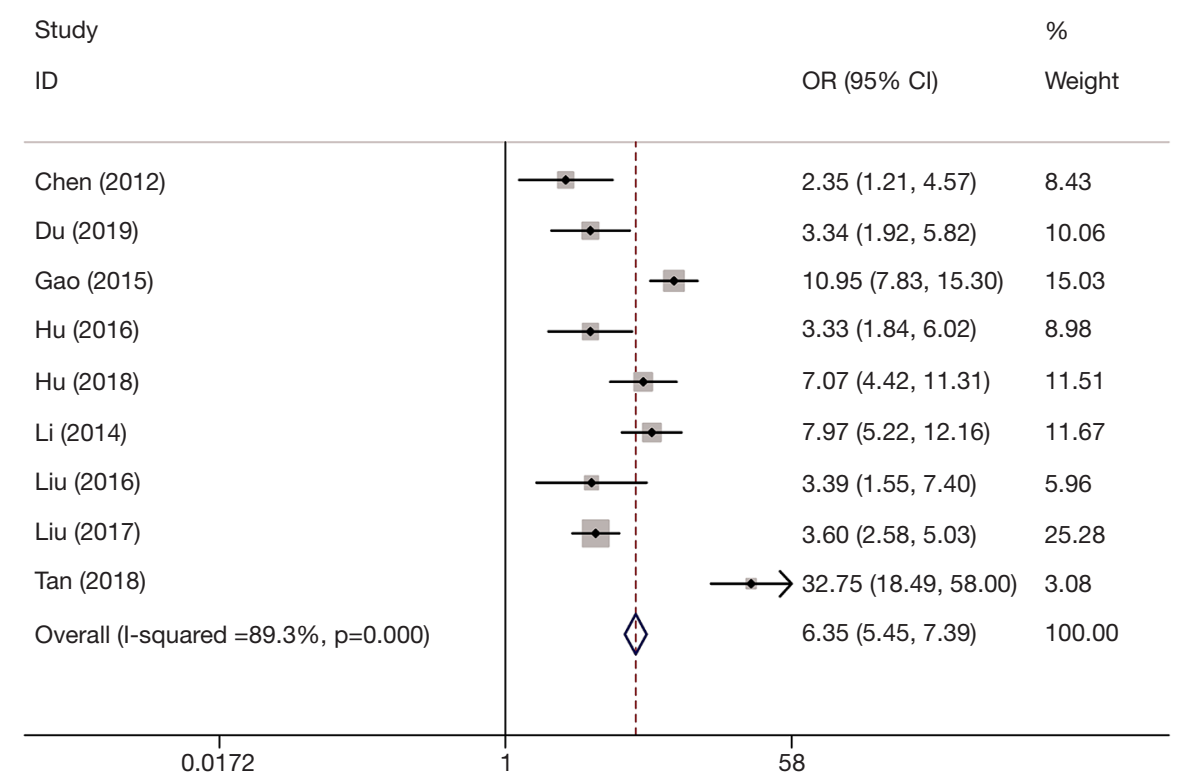

Figure 4 The forest plot for the predictive value of NLR for prognosis in patients with HCC. NLR, neutrophil-to-lymphocyte ratio; HCC, hepatocellular carcinoma.

Table 3 Subgroup analysis on the basis of different standards

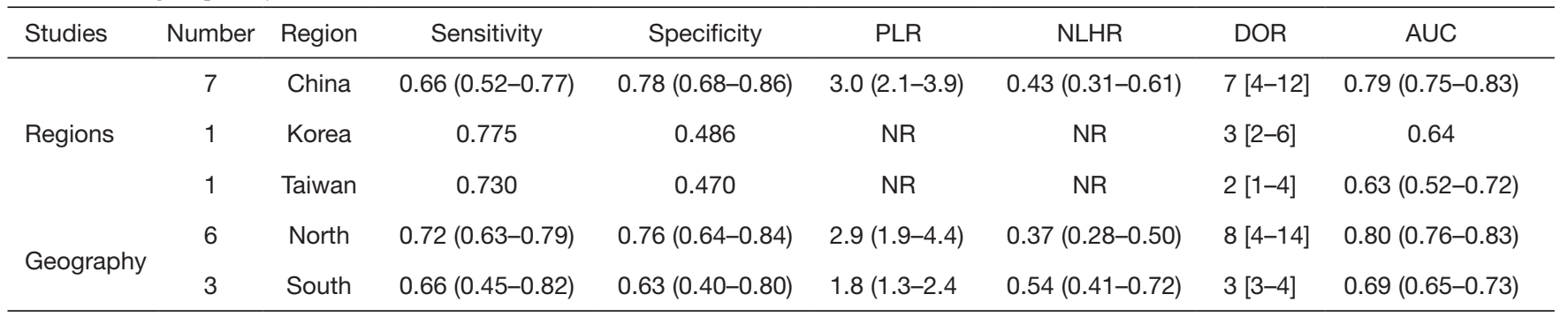

NR, no result; PLR, positive likelihood ratio; NLHR, negative likelihood ratio; DOR, diagnostic odds ratio; AUC, area under the curve.

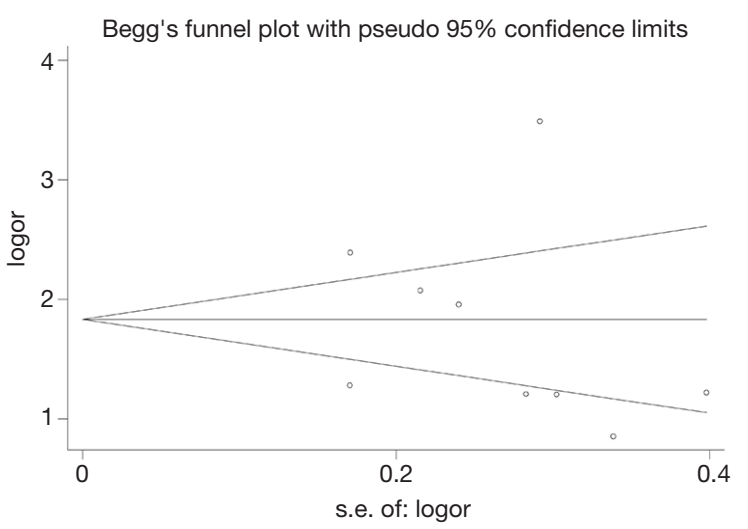

Figure 5 Begg's funnel plot for testing publication bias. and HCC prognosis. The pooled outcomes in these cohorts indicated that high baseline NLR was a remarkable predictor of poor prognosis of HCC patients, indicating that NLR is a valuable inflammatory biomarker with high sensitivity, specificity, and DOR. In addition, subgroup analyses indicated that NLR worked better as a prognostic predictor in HCC patients from mainland China than in those from Korea and Taiwan. Moreover, high baseline NLR also significantly correlated with the prognostic value geographically, indicating that NLR exhibits a better prognostic role in the North than in the South of East Asia.

Generally, high baseline NLR reflects local and systemic 
inflammation, which forms a favorable microenvironment to promote tumor invasion and metastasis (43). Previous studies reported that NLR not only reflects a tumor-friendly microenvironment but also systemic immune status, which both benefits tumor invasion and restrains host immune surveillance (44-46). It is worth noting that in addition to NLR, other laboratory markers of systemic inflammatory response have been reported to play a prognostic role in cancer patients, such as modified Glasgow prognostic score $(47,48)$, and notably $\mathrm{C}$ reactive protein (CRP) (49). Furthermore, biological markers $(50,51)$ and gene polymorphisms (52) have also been suggested as prognostic indicators in cancer patients. Nevertheless, NLR stands out with its low cost, broad practicality, and accessibility (18). The pooled results of our meta-analysis encourage routine monitoring of NLR for poor prognosis and recurrence of HCC patients, regardless of tumor stage and geographic region.

In our subgroup analysis, a better prognostic value was observed in HCC patients from mainland China (DOR, 7; AUC, 0.79) than in those from both Korea (DOR, 3; AUC, 0.64) and Taiwan (DOR, 2; AUC, 0.63). This may be explained by the fact that HCC is the second most common cancer in mainland China, indicating that HCC is more severe in China than the other 2 regions (53). For subgroup geographic analysis, a better prognostic performance of NLR as a predictor of prognosis was seen in HCC patients from the North (DOR, 8; AUC, 0.80) than in those from the South (DOR, 3; AUC, 0.69). This may be related to differences in the dietary habits and lifestyles of people in the North versus the South, as well as climate differences (54). Interestingly, it has been indicated that the incidence of HCC is influenced by differences in habits between northern and the southern populations, and people living in the South may have a relatively low risk of HCC.

Subsequently, meta-regression and interaction revisited subgroup analyses were adopted to determine the causes of heterogeneity observed among the included studies. Several stratified analyses concerning region or geography were performed to investigate the sources of heterogeneity. Moreover, the outcome of any single study as a main source of heterogeneity was ruled out by performing leave-one-out sensitivity analysis. The pooled results indicated that the main source of heterogeneity stemmed from geographical characteristics (North: $\mathrm{I}^{2}=88.8 \%, \mathrm{P} \leq 0.001$ or South: $\mathrm{I}^{2}=0$, $\mathrm{P}=0.532$ ). However, the region (mainland China: $\mathrm{I}^{2}=90.1 \%$, $\mathrm{P} \leq 0.001$, Korea or Taiwan) was not found to be a source of heterogeneity in the predictive value of NLR. It has been consistently found that differences in environmental factors, population characteristics, lifestyle, and sample sizes are the main sources of heterogeneity (55). Further verification of the above conclusions needs to be achieved by incorporating more studies that conform to the inclusion criteria.

Several limitations in our study should also be mentioned. First, the literature search was restricted to open-access studies. However, relevant studies that were not readily available might also have met the inclusion criteria of our meta-analysis but were inevitably excluded. Second, heterogeneity was observed in this study because of confounding factors, including the cut-off value of NLR and sample size. In spite of this, meta-regression and subgroup analyses eliminated the need to fully explaining the heterogeneity by both of the above-mentioned confounders. Third, the correlation between elevated NLR and clinicopathological parameters of patients, including tumor stage and differentiation grade, was not analyzed because of insufficient data for analysis or lack of relevant information in some of the enrolled studies. Lastly, the association between high baseline NLR and poor prognosis of HCC was demonstrated in a majority of original studies, which may be explained by an easily accessible publication of positive results, ultimately leading difficulty in locating more controversial studies.

\section{Conclusions}

In conclusion, the present meta-analysis revealed a significant association between high baseline NLR and poor prognosis in HCC patients, especially in those from East Asian regions with high incidence of HCC. Therefore, NLR may be used as an inflammatory factor for efficient evaluation of prognosis of HCC, which can be useful in determining individual treatment designs and stratifying patients. Even so, larger-scale and more well-designed investigations are warranted to further elucidate the prognostic value of NLR in HCC patients.

\section{Acknowledgments}

The authors thank the study participants in each of the individual studies for their involvement.

Funding: This study was supported by the National Natural Science Foundation of China (No. 81803652, 31871244 and 81973733), the Natural Science Foundation of Guangdong Province (No. 2019A1515011555 and 2018A0303100007), the Shenzhen Foundation of Health and Family Planning Commission (No. SZBC2018016), and the Special Fund for 
Economic and Technological Development of Longgang District of Shenzhen City (No. LGKCYLWS2019000361 and LGKCYLWS2020064).

\section{Footnote}

Reporting Checklist: The authors have completed the PRISMA reporting checklist. Available at http://dx.doi. org/10.21037/tcr-20-3237

Conflicts of Interest: All authors have completed the ICMJE uniform disclosure form (available at http://dx.doi. org/10.21037/tcr-20-3237). The authors have no conflicts of interest to declare.

Ethical Statement: The authors are accountable for all aspects of the work in ensuring that questions related to the accuracy or integrity of any part of the work are appropriately investigated and resolved.

Open Access Statement: This is an Open Access article distributed in accordance with the Creative Commons Attribution-NonCommercial-NoDerivs 4.0 International License (CC BY-NC-ND 4.0), which permits the noncommercial replication and distribution of the article with the strict proviso that no changes or edits are made and the original work is properly cited (including links to both the formal publication through the relevant DOI and the license). See: https://creativecommons.org/licenses/by-nc-nd/4.0/.

\section{References}

1. Gutierrez JA, Gish RG. Efficacy of combination treatment modalities for intermediate and advanced hepatocellular carcinoma: intra-arterial therapies, sorafenib and novel small molecules. Transl Cancer Res 2013;2:460-71.

2. Feng K, Yan J, Li X, et al. A randomized controlled trial of radiofrequency ablation and surgical resection in the treatment of small hepatocellular carcinoma. J Hepatol 2012;57:794-802.

3. Song IH, Kim KS. Current status of liver diseases in Korea: hepatocellular carcinoma. Korean J Hepatol 2009;15 Suppl 6:S50-9.

4. Wang FS, Fan JG, Zhang Z, et al. The global burden of liver disease: the major impact of China. Hepatology 2014;60:2099-108.

5. Arizumi T, Ueshima K, Iwanishi M, et al. The Overall Survival of Patients with Hepatocellular Carcinoma
Correlates with the Newly Defined Time to Progression after Transarterial Chemoembolization. Liver Cancer 2017;6:227.

6. Fan ST, Lo CM, Liu CL, et al. Hepatectomy for hepatocellular carcinoma: toward zero hospital deaths. Ann Surg 1999;229:322-30.

7. Maluccio M, Covey A. Recent progress in understanding, diagnosing, and treating hepatocellular carcinoma. CA Cancer J Clin 2012;62:394-9.

8. Poon RT, Fan ST, Ng IO, et al. Different risk factors and prognosis for early and late intrahepatic recurrence after resection of hepatocellular carcinoma. Cancer 2000;89:500-7.

9. Imamura H, Matsuyama $\mathrm{Y}$, Tanaka E, et al. Risk factors contributing to early and late phase intrahepatic recurrence of hepatocellular carcinoma after hepatectomy. J Hepatol 2003;38:200-7.

10. Jemal A, Bray F, Center MM, et al. Global cancer statistics. CA Cancer J Clin 2011;61:69-90.

11. Motomura T, Shirabe K, Mano Y, et al. Neutrophillymphocyte ratio reflects hepatocellular carcinoma recurrence after liver transplantation via inflammatory microenvironment. J Hepatol 2013;58:58-64.

12. Azab B, Bhatt VR, Phookan J, et al. Usefulness of the neutrophil-to-lymphocyte ratio in predicting short- and long-term mortality in breast cancer patients. Ann Surg Oncol 2012;19:217-24.

13. Chua W, Charles KA, Baracos VE, et al. Neutrophil/ lymphocyte ratio predicts chemotherapy outcomes in patients with advanced colorectal cancer. Br J Cancer 2011;104:1288-95.

14. Fu SJ, Shen SL, Li SQ, et al. Prognostic value of preoperative peripheral neutrophil-to-lymphocyte ratio in patients with $\mathrm{HBV}$-associated hepatocellular carcinoma after radical hepatectomy. Med Oncol 2013;30:721.

15. Yao Y, Yuan D, Liu H, et al. Pretreatment neutrophil to lymphocyte ratio is associated with response to therapy and prognosis of advanced non-small cell lung cancer patients treated with first-line platinum-based chemotherapy. Cancer Immunol Immunother 2013;62:471-9.

16. Stotz M, Gerger A, Eisner F, et al. Increased neutrophillymphocyte ratio is a poor prognostic factor in patients with primary operable and inoperable pancreatic cancer. Br J Cancer 2013;109:416-21.

17. Zahorec R. Ratio of neutrophil to lymphocyte counts-rapid and simple parameter of systemic inflammation and stress in critically ill. Bratisl Lek Listy 2001;102:5-14.

18. Hu K, Lou L, Ye J, et al. Prognostic role of the neutrophil- 
lymphocyte ratio in renal cell carcinoma: a meta-analysis. BMJ Open 2015;5:e006404.

19. Xiao WK, Chen D, Li SQ, et al. Prognostic significance of neutrophil-lymphocyte ratio in hepatocellular carcinoma: a meta-analysis. BMC Cancer 2014;14:117.

20. Liao W, Zhang J, Zhu Q, et al. Preoperative Neutrophilto-Lymphocyte Ratio as a New Prognostic Marker in Hepatocellular Carcinoma after Curative Resection 1. Transl Oncol 2014;7:248-55.

21. Mano Y, Shirabe K, Yamashita Y, et al. Preoperative neutrophil-to-lymphocyte ratio is a predictor of survival after hepatectomy for hepatocellular carcinoma: a retrospective analysis. Ann Surg 2013;258:301-5.

22. Kishi Y, Kopetz S, Chun YS, et al. Blood neutrophilto-lymphocyte ratio predicts survival in patients with colorectal liver metastases treated with systemic chemotherapy. Ann Surg Oncol 2009;16:614-22.

23. Najjar M, Agrawal S, Emond JC, et al. Pretreatment neutrophil-lymphocyte ratio: useful prognostic biomarker in hepatocellular carcinoma. J Hepatocell Carcinoma 2018;5:17-28.

24. Moher D, Liberati A, Tetzlaff J, et al. Preferred reporting items for systematic reviews and meta-analyses: the PRISMA statement. Revista Española De Nutrición Humana Y Dietética 2009;18:e123.

25. McInnes MDF, Moher D, Thombs BD, et al. Preferred Reporting Items for a Systematic Review and Metaanalysis of Diagnostic Test Accuracy Studies: The PRISMA-DTA Statement. JAMA 2018;319:388-96.

26. Higgins JP, Altman DG, Gotzsche PC, et al. The Cochrane Collaboration's tool for assessing risk of bias in randomised trials. BMJ 2011;343:d5928.

27. Stang A. Critical evaluation of the Newcastle-Ottawa scale for the assessment of the quality of nonrandomized studies in meta-analyses. Eur J Epidemiol 2010;25:603-5.

28. Wu H, Zhang H, Li P, et al. Association between dietary carbohydrate intake and dietary glycemic index and risk of age-related cataract: a meta-analysis. Invest Ophthalmol Vis Sci 2014;55:3660-8.

29. Dong F, Yang G, Pan HW, et al. The association of PTPN22 rs2476601 polymorphism and CTLA-4 rs231775 polymorphism with LADA risks: a systematic review and meta-analysis. Acta Diabetologica 2014;51:691-703.

30. Xia C, Amador C, Huffman J, et al. Pedigree- and SNP-Associated Genetics and Recent Environment are the Major Contributors to Anthropometric and Cardiometabolic Trait Variation. Plos Genetics 2016;12:e1005804.
31. Macdonald SPJ, Stone SF, Neil CL, et al. Sustained elevation of resistin, NGAL and IL- 8 are associated with severe sepsis/septic shock in the emergency department. PLoS One 2014;9:e110678.

32. Cantoni V, Green R, Acampa W, et al. Long-term prognostic value of stress myocardial perfusion imaging and coronary computed tomography angiography: A metaanalysis. J Nucl Cardiol 2016;23:185-97.

33. Begg CB, Mazumdar M. Operating characteristics of a rank correlation test for publication bias. Biometrics 1994;50:1088-101.

34. Du Z, Dong J, Bi J, et al. Correction: Predictive value of the preoperative neutrophil-to-lymphocyte ratio for the development of hepatocellular carcinoma in HBVassociated cirrhotic patients after splenectomy. PLoS One 2018;13:e0195336.

35. Gao F, Li X, Geng M, et al. Pretreatment NeutrophilLymphocyte Ratio: An Independent Predictor of Survival in Patients With Hepatocellular Carcinoma. Medicine 2015;94:e639.

36. Hu J, Wang N, Yang Y, et al. Diagnostic value of alphafetoprotein combined with neutrophil-to-lymphocyte ratio for hepatocellular carcinoma. BMC Gastroenterol 2018;18:018-0908.

37. Li X, Han Z, Cheng Z, et al. Preoperative neutrophil-tolymphocyte ratio is a predictor of recurrence following thermal ablation for recurrent hepatocellular carcinoma: a retrospective analysis. PLoS One 2014;9:e110546.

38. Liu Y, Wang ZX, Cao Y, et al. Preoperative inlfammationbased markers pre-dict early and late recurrence of hepatocellular carcinoma after curative hepatectomy. Hepatobiliary \& Pancreatic Diseases International Hbpd Int 2016;15:266-74.

39. Liu C, Jia BS, Zou BW, et al. Neutrophil-to-lymphocyte and aspartate-to-alanine aminotransferase ratios predict hepatocellular carcinoma prognosis after transarterial embolization. Medicine 2017;96:e8512.

40. Tan W, Sun W, Li X, et al. Preablation neutrophil-tolymphocyte ratio as an independent prognostic factor in locally advanced hepatocellular carcinoma patients following radiofrequency ablation. J Cancer Res Ther 2018;14:84-9.

41. Hu XG, Mao W, Park YK, et al. Blood Neutrophil-toLymphocyte Ratio Predicts Tumor Recurrence in Patients with Hepatocellular Carcinoma within Milan Criteria after Hepatectomy. Yonsei Med J 2016;57:1115-23.

42. Chen TM, Lin CC, Huang PT, et al. Neutrophil-tolymphocyte ratio associated with mortality in early 
hepatocellular carcinoma patients after radiofrequency ablation. J Gastroenterol Hepatol 2012;27:553-61.

43. Grivennikov SI, Greten FR, Karin M. Immunity, inflammation, and cancer. Cell 2010;140:883-99.

44. Schreiber RD, Old LJ, Smyth MJ. Cancer immunoediting: integrating immunity's roles in cancer suppression and promotion. Science 2011;331:1565-70.

45. Mano Y, Shirabe K, Yamashita Y, et al. Preoperative neutrophil-to-lymphocyte ratio is a predictor of survival after hepatectomy for hepatocellular carcinoma: a retrospective analysis. Ann Surg 2013;258:301-5.

46. Castelli G, Pelosi E, Testa U. Liver Cancer: Molecular Characterization, Clonal Evolution and Cancer Stem Cells. Cancers 2017;9:127.

47. Lamb GW, Aitchison M, Ramsey S, et al. Clinical utility of the Glasgow Prognostic Score in patients undergoing curative nephrectomy for renal clear cell cancer: basis of new prognostic scoring systems. Br J Cancer 2012;106:279-83.

48. Tai CG, Johnson TV, Abbasi A, et al. External validation of the modified Glasgow prognostic score for renal cancer. Indian J Urol 2014;30:33-7.

49. Jagdev SP, Gregory W, Vasudev NS, et al. Improving the accuracy of pre-operative survival prediction in renal

Cite this article as: Lin S, Hu S, Ran Y, Wu F. Neutrophilto-lymphocyte ratio predicts prognosis of patients with hepatocellular carcinoma: a systematic review and metaanalysis. Transl Cancer Res 2021;10(4):1667-1678. doi: 10.21037/ tcr-20-3237 cell carcinoma with C-reactive protein. Br J Cancer 2010;103:1649-56.

50. Vilella-Arias SA, Rocha RM, Da CW, et al. Loss of caspase 7 expression is associated with poor prognosis in renal cell carcinoma clear cell subtype. Urology 2013;82:974.e1-e7.

51. Shi X, Jiang J, Ye X, et al. Prognostic prediction and diagnostic role of intercellular adhesion molecule-1 (ICAM1) expression in clear cell renal cell carcinoma. J Mol Histol 2014;45:427-34.

52. de Martino M, Haitel A, Schatzl G, et al. The protease activated receptor 1 gene variation IVSn $-14 \mathrm{~A}>\mathrm{T}$ is associated with distant metastasis and cancer specific survival in renal cell carcinoma. J Urol 2013;190:1392-7.

53. Fu J, Wang $\mathrm{H}$. Precision diagnosis and treatment of liver cancer in China. Cancer Letters 2018;412:283.

54. Zeng Y, Du J, Pu X, et al. Coevolution between cancer activities and food structure of human being from Southwest China. BioMed Research International 2015;2015:497934.

55. Nansseu JRN, Ngoum SS, Balti EV. Incidence, prevalence and genetic determinants of neonatal diabetes mellitus: a systematic review and meta-analysis protocol. Syst Rev 2016;5:188. 